\title{
KVG: Packen wir es nun an!
}

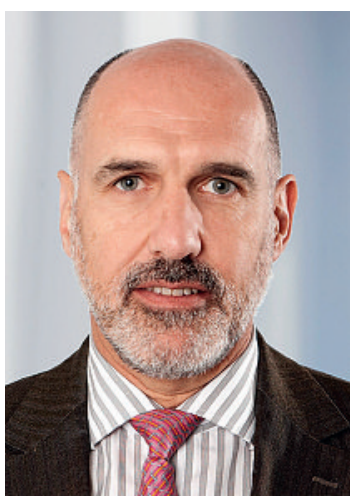

Die KVG-Revision im Bereich ambulante Medizin ist in den letzten Monaten mit grossen Schritten vorangekommen; zumeist gehen diese Schritte in die richtige Richtung. Nun müssen die letzten noch hängigen Fragen geklärt werden, ohne dass unnötige Verzögerungen beziehungsweise immer wieder auftretende Ausweichmanöver zugelassen werden

Einer der letzten Punkte, die noch zu regeln sind, ist der Risikoausgleich, der zurzeit nicht befriedigend funktioniert, was zur bekannten «Jagd auf gute Risiken» sowie zu unangemessenen «Wirtschaftlichkeitsverfahren» der Versicherer gegen einige Kollegen führt: Teure Patienten sind ein Problem, das bisher überhaupt nicht gelöst ist. Sobald die integrierten Versorgungsnetze breit eingeführt werden, wird zudem ein verfeinerter Risikoausgleich unabdingbar sein, damit sich die Versicherer konstruktiv an dieser Einführung beteiligen.

Ab 2012 ist zwar eine Verbesserung des Risikoausgleichs vorgesehen, doch diese ist unzureichend oder gar unangemessen und erfolgt verspätet.

Diesen Punkt möchte ich gerne genauer ausführen:

Zunächst ist auf die Situation der chronischkranken Patienten hinzuweisen. Ihnen bietet Managed Care den höchsten Nutzen. Denn am meisten bringt die «integrierte» Betreuung auf die Dauer. Zudem können die Netzwerke Leistungen anbieten und übernehmen, die nicht obligatorisch sind, deren Nutzen jedoch offensichtlich ist (zum Beispiel Ernährungsberatung oder Fusspflege für Diabetiker). Diese kohärente oder gar erweiterte Betreuung wird sich längerfristig «auszahlen», sowohl für die betreffenden Patienten als auch für das Gesundheitssystem.

\section{Ab Inkrafttreten der KVG-Revision muss \\ unbedingt ein verfeinerter Risikoausgleich vorliegen, der von der Morbidität ausgeht}

Damit die Ziele von Managed Care erreicht werden können, müssen aufwendige Patienten deshalb unbedingt dazu bewegt werden, sich an Managed Care zu beteiligen. Sie müssen zum einen durch die angebotenen Leistungen und deren Qualität angezogen werden. Vor allem aber dürfen sie nicht mehr von den Versicherern oder gar den Netzwerken zurückgewiesen werden, weil sie das Budget belasten. Diese Patienten müssen attraktiv werden! Ab Inkrafttreten der KVGRevision muss deshalb unbedingt ein verfeinerter Risikoausgleich vorliegen, der von der Morbidität ausgeht (d.h. von der wirtschaftlichen Belastung, die durch die Krankheiten verursacht wird).

Ein weiteres Beispiel sind die Spitalaufenthalte. Über das Alter und Geschlecht der Versicherten hinaus bilden die Spitalaufenthalte das neue Kriterium für den Risikoausgleich, das ab 2012 zu berücksichtigen ist: Ein Spitalaufenthalt hat zur Folge, dass der Patient der Kategorie «teurer Patient» zugeordnet und ein Ausgleich gewährt wird.

\section{Obwohl die Vorlage nicht vollumfäng- lich befriedigt, entspricht die vor- gesehene KVG-Revision in zahlreichen Punkten den Wünschen, die die FMH und namentlich die Ärztekammer
seit 2006 geäussert haben}

Das ist ein typisches Beispiel für eine nur auf den ersten Blick gute Idee, für einen gefährlichen Anreiz! Denn in Wirklichkeit ist es nicht nur für die Patienten, sondern auch für das Gesundheitssystem viel besser, aufwendige Patienten, zum Beispiel Herz- oder Krebspatienten, möglichst lange ambulant zu behandeln. Im vorgesehenen System werden diese ambulanten Patienten jedoch nicht als teuer anerkannt, und es wird somit kein Ausgleich gewährt ... deshalb würde es sich empfehlen, sie ins Spital einzuweisen. Auch in dieser Hinsicht sollte der Risikoausgleich so rasch als möglich verfeinert werden.

Daher müssen die «Morbiditätskriterien» im Risikoausgleich rasch berücksichtigt werden. Es darf nicht einfach zugewartet werden, was geschehen wird, wenn nach 2012 die Spitalaufenthalte berücksichtigt werden - die Morbiditätskriterien müssen jetzt im Rahmen der laufenden Revision in den Risikoausgleich aufgenommen werden. Unser Vizepräsident, Nationalrat Ignazio Cassis, hat bereits einen entsprechenden Änderungsantrag gestellt, der Unterstützung verdient!

Nun muss diese Revision als Gesamtpaket vorangetrieben werden. Bereits zeichnen sich Blockierungsversuche ab, indem versucht wird, Elemente in die Vorlage einzubringen, die ein Referendum auslösen würden - ob die immer wieder aufgewärmte Vertragsfreiheit oder die neuere Idee, Versicherte, die eine Prämienverbilligung erhalten, zu Managed Care zu verpflichten. Diese Manöver sind inakzeptabel.

Obwohl die Vorlage nicht vollumfänglich befriedigt, entspricht die vorgesehene KVG-Revision in zahlreichen Punkten den Wünschen, die die FMH und namentlich die Ärztekammer seit 2006 geäussert haben. Wir hoffen, dass diese Vorlage nun rasch unter Dach und Fach gebracht werden kann. Mit einem verfeinerten Risikoausgleich!

Dr. med. Jacques de Haller, Präsident der FMH 